\title{
Scientific basis of biomarkers and benefits of functional foods for reduction of disease risk: cancer
}

\author{
Joseph J. Rafter* \\ Department of Medical Nutrition, Karolinska Institutet, NOVUM, S-141 86 Huddinge, Sweden
}

\begin{abstract}
One of the most promising areas for the development of functional foods lies in modification of the activity of the gastrointestinal tract by use of probiotics, prebiotics and synbiotics. While a myriad of healthful effects have been attributed to the probiotic lactic acid bacteria, perhaps the most controversial remains that of anticancer activity. However, it must be emphasised that, to date, there is no direct experimental evidence for cancer suppression in man as a result of consumption of lactic cultures in fermented or unfermented dairy products, although there is a wealth of indirect evidence, based largely on laboratory studies. Presently, there are a large number of biomarkers available for assessing colon cancer risk in dietary intervention studies, which are validated to varying degrees. These include colonic mucosal markers, faecal water markers and immunological markers. Overwhelming evidence from epidemiological, in vivo, in vitro and clinical trial data indicates that a plant-based diet can reduce the risk of chronic disease, particularly cancer. It is now clear that there are components in a plant-based diet other than traditional nutrients that can reduce cancer risk. More than a dozen classes of these biologically active plant chemicals, now known as 'phytochemicals', have been identified. Although the vast number of naturally occurring health-enhancing substances appear to be of plant origin, there are a number of physiologically active components in animal products (such as the probiotics referred to above) that deserve attention for their potential role in cancer prevention.
\end{abstract}

Probiotics: Cancer: Biomarkers: Phytochemicals: Animal products

\section{Introduction}

In recent years, there has been a growing interest in the concept of 'functional foods' by both the food industry and the consumer. Functional foods are defined as foodstuffs that improve overall health and/or reduce the risk of disease. Thus, with the consumer becoming more interested in foods which benefit health and the food industry beginning to understand the market potential of functional foods, solid research activity in the area has been initiated world-wide. In addition, when one considers the costs to society of diseases such as obesity, cardiovascular disease, diabetes, food allergies, osteoporosis and cancer, all of which may be influenced by diet, the potential of wellcharacterised functional foods becomes even more evident.

Although a large number of naturally occurring healthenhancing substances are of plant origin, there are a number of physiologically active components in animal products that deserve attention for their potential role in disease prevention.

One such example of a functional food, which has been the focus of increasing research activity in recent years, are probiotics: live microbial feed supplements that beneficially affect the host animal by improving its intestinal microbial balance (Fuller, 1989). Probiotics usually refer to highly selected lactic acid bacteria, e.g. Lactobacillus spp., Bifidobacterium spp. and Streptococcus spp., with defined gut survival properties and associated biological activities, that can be ingested in fermented milk products or as a supplement. The list of healthful effects attributed to probiotic bacteria is extensive and includes alleviation of lactose intolerance symptoms, serum cholesterol reduction, anticancer effects, alleviating constipation and relieving vaginitis, to name but a few. The vast majority of studies on the anticancer effects deal with colorectal cancer (Hirayama \& Rafter, 2000), although there are some on breast and bladder cancer.

There is also a large amount of evidence, from epidemiological, in vivo, in vitro and clinical trial data, indicating that a plant-based diet can reduce the risk of chronic disease, particularly cancer. In 1992, a review of 200 epidemiological studies showed that cancer risk in 
people consuming diets high in fruits and vegetables was only one-half that in those consuming few of these foods (Block et al. 1992). It is now clear that there are components in a plant-based diet other than traditional nutrients that can reduce cancer risk. More than a dozen classes of these biologically active plant chemicals, now known as 'phytochemicals', have been identified. Several examples are given below.

\section{Functional foods from animal sources}

\section{Dairy products}

Epidemiological studies regarding the effect of milk and dairy products on colorectal cancer incidence provide conflicting results. Some studies report a negative association between consumption of dairy products and disease incidence, whereas others report no or even a positive association. One reason for this discrepancy in the epidemiological observations may lie in the fact that some dairy products, such as cheese and cream, contain large amounts of animal fat, which is considered a risk factor by some for colorectal cancer. In line with this assumption, it appeared that the positive correlation between dairy products and colorectal cancer found in one study was due to cheese consumption (Iscovich et al. 1992). In addition, an international correlation study observed a negative correlation between consumption of dairy products and colorectal cancer only after adjustment for animal fat intake (McKeown-Eyssen \& Bright-See, 1984). Most of the studies that reported no association did not adjust for fat intake. Hence, epidemiological studies provide weak evidence that consumption of milk and dairy products could protect against colorectal cancer.

The main hypothesis to explain this effect has been the high $\mathrm{Ca}$ content of dairy products. Thus, many previous studies have focused on $\mathrm{Ca}$ and consequently examined the effects of dietary Ca supplements (Lapre et al. 1993) or Ca-deprived milk (Govers et al. 1996) on risk markers for the disease, in controlled dietary intervention studies. While the results of these studies generally do show a protective effect of $\mathrm{Ca}$ on the biomarkers, this is not always the case. However, recently, a great deal of research has been dealing with other components in dairy products, particularly fermented dairy products, i.e. the probiotic bacteria.

Many studies confirm the involvement of the endogenous microflora in the onset of colon cancer. This makes it reasonable to think that changing the intestinal microflora could influence tumour development. Thus, considerable attention has focused on dietary components that can influence the gut microflora as a strategy for colon cancer prevention. In particular, evidence has been accumulating from animal studies for a protective role for probiotics, prebiotics (non-digestible carbohydrates that stimulate lactic acid bacteria numbers in the gut) and synbiotics (combinations of pro- and prebiotics).

Several studies have indicated that addition of probiotic cultures (e.g. Lactobacillus rhamnosus GG, Lactobacillus salivarius or Bifidobacterium longum) to the diet of rats treated with colon carcinogens reduces colon tumour incidence and multiplicity (Hirayama \& Rafter, 2000). The precise mechanisms by which lactic acid bacteria may inhibit colon cancer are presently unknown. However, such mechanisms might include: enhancing the host's immune response; binding and degrading potential carcinogens; quantitative and/or qualitative alterations in the intestinal microflora incriminated in producing putative carcinogen(s) and promoters (e.g. bile acid-degrading bacteria); producing anti-tumourigenic or anti-mutagenic compounds in the colon; alteration of the metabolic activities of intestinal microflora; alteration of physicochemical conditions in the colon; and effects on the physiology of the host.

A wide range of studies have also been conducted in rodent models, including transgenic mice, on the cancermodulating effect of dietary prebiotics such as fructooligosaccharides, inulins and lactulose. These have consistently demonstrated a reduction in the endpoints measured - colonocyte DNA damage, aberrant crypt foci in the colon (believed to be pre-neoplastic lesions), number of tumours per animal, size and growth rate of the tumours, mean survival time, increase in life span (Pierre, 1997). In all animal experiments where mixtures of probiotics (Bifidobacterium spp., Lactobacillus casei, L. rhamnosus) and prebiotics (lactulose, long-chain inulin, short-chain fructo-oligosaccharides) were tested, the effect of the mixture on the measured biomarkers was greater than the sum of the two separately, suggesting a combinational advantage of synbiotics.

However, to date, there is no definite evidence that probiotics actually reduce the risk of human colon cancer. The epidemiological studies are inconsistent (Hirayama \& Rafter, 2000). Consumption of large quantities of dairy products such as yoghurt and fermented milk containing Lactobacillus or Bifidobacterium may be related to a lower incidence of colon cancer. An epidemiological study performed in Finland demonstrated that, despite the high fat intake, colon cancer incidence was lower than in other countries because of the high consumption of milk, yoghurt and other dairy products (Intestinal Microbiology Group, 1977). In two population-based case-control studies of colon cancer, an inverse association was observed for yoghurt (Peters et al. 1992) and cultured milk (Young \& Wolf, 1988) consumption, adjusted for potential confounding variables. It can also be mentioned that an inverse relationship has been demonstrated between the frequency of consumption of yoghurt and other fermented milk products and breast cancer in women (van't Veer et al. 1989). On the other hand, two companion American prospective studies, the 1980-1988 follow-up of the Nurses' Health Study and the 19861990 follow-up of the Health Professionals' Follow-up Study, did not provide evidence that intake of dairy products is associated with a decreased risk of colon cancer (Kampman et al. 1994a). In a cohort study in The Netherlands, it was shown that the intake of fermented dairy products was not significantly associated with colorectal cancer risk in an elderly population with a relatively wide variation in dairy product consumption, although a weak non-significant inverse association with colon cancer was observed (Kampman et al. 1994b). 
In addition, there are some studies examining the effect of probiotics on the biomarkers for colon cancer risk (see below) in healthy volunteers and patients. Consumption of lactic acid bacteria by volunteers has been shown to reduce the mutagenicity of urine and faeces associated with the ingestion of carcinogens in cooked meat (Lidbeck et al. 1992). Mucosal cell proliferative activity in upper colonic crypts of patients with colon adenomas (believed to be a risk factor for tumour development) decreased significantly after the administration of Lactobacillus acidophilus and Bifidobacterium bifidus cultures (Biasco et al. 1991).

\section{Beef}

An anticarcinogenic fatty acid known as conjugated linoleic acid was first isolated from grilled beef in 1987 (Ha et al. 1987). Nine different isomers of conjugated linoleic acid have been reported as occurring naturally in food. Conjugated linoleic acid is unique in that it is found in highest concentrations in fat from ruminant animals (e.g. beef, dairy, lamb). In recent years, conjugated linoleic acid has been shown to be effective in suppressing forestomach tumours in mice, aberrant colonic crypt foci in rats and mammary tumours in rats (Ip \& Scimeca, 1997).

\section{Functional foods from plant sources}

\section{Soyabeans}

Several classes of anticarcinogens have been identified in soyabeans, including protease inhibitors, phytosterols, saponins, phenolic acids, phytic acid and isoflavones. Of these, isoflavones (genistein and daidzein) are particularly noteworthy because soyabeans are the only significant dietary source of these compounds. Isoflavones are heterocyclic phenols structurally similar to the oestrogenic steroids. Because they are weak oestrogens, isoflavones may act as anti-oestrogens by competing with the naturally occurring endogenous oestrogens. This may explain why populations that consume significant amounts of soya have reduced risk of oestrogen-dependent cancer. However, more epidemiological data and clinical intervention trials are needed to investigate the role of soya in reducing cancer risk.

\section{Flaxseed}

There has been an increasing interest in fibre-associated compounds known as lignans. The two primary mammalian lignans, enterodiol and its oxidation product, enterolactone, are formed in the intestinal tract by bacterial action on plant lignan precursors. Flaxseed is one of the richest sources of mammalian lignan precursors. Because enterodiol and enterolactone are structurally similar to both naturally occurring and synthetic oestrogens, and have been shown to possess weakly oestrogenic and anti-oestrogenic activities, they may also play a role in the prevention of oestrogen-dependent cancers. In rodents, flaxseed has been shown to decrease tumours of the colon, mammary gland and lung. Phipps et al. (1993) demon- strated that the ingestion of $10 \mathrm{~g}$ of flaxseed per day elicited several hormonal changes associated with reduced breast cancer risk. However, as is the case with soya, epidemiological data are required to support the hypothesis that enterodiol and enterolactone have anticarcinogenic properties in man.

\section{Tomatoes}

Tomatoes have received much attention in recent years because of interest in lycopene, the primary carotenoid in this fruit, and its potential role in cancer risk reduction (Weisburger, 1998). In a prospective cohort study of more than 47000 men, those who consumed tomato products ten or more times per week had less than onehalf the risk of developing advanced prostate cancer (Giovannucci et al. 1995). Interestingly, lycopene is the most abundant carotenoid in the prostate gland. Other cancers whose risk have been inversely associated with serum or tissue levels of lycopene include breast, digestive tract, cervix, bladder and skin (Clinton, 1998). Proposed mechanisms by which lycopene could influence cancer risk are related to its antioxidant function. Lycopene is the most efficient quencher of singlet oxygen in biological systems (Di Mascio et al. 1989).

\section{Garlic}

Garlic (Allium sativum) is probably the herb most widely quoted in the literature for medicinal purposes. The intact garlic bulb contains an odourless amino acid, which is converted enzymatically by allinase into allicin when the garlic cloves are crushed (Block, 1992). Allicin then decomposes spontaneously to form numerous sulfurcontaining compounds, some of which have been investigated for their chemopreventive activity. Garlic components have been shown to inhibit tumourigenesis in several experimental models. However, additional reports have shown garlic to be ineffective. Inconclusive results are likely to be due to differences in the type of garlic compounds or preparations used by various investigators. Several epidemiological studies show that garlic may be effective in reducing human cancer risk (Dorant et al. 1993). However, it should be mentioned that not all such studies have shown garlic to be protective. A review of twenty epidemiological studies (Ernst, 1997) suggested that allium vegetables, including onions, may confer a protective effect on cancers of the gastrointestinal tract.

\section{Tea}

Much attention has focused on the polyphenolic constituents of tea, particularly green tea. Polyphenols comprise up to $30 \%$ of the total dry weight of fresh tea leaves. Catechins are the predominant and most significant of all tea polyphenols (Graham, 1992). The four major green tea catechins are epigallocatechin-3-gallate, epigallocatechin, epicatechin-3-gallate and epicatechin. Much of the work on the health effects of tea has focused on its cancer chemopreventive effects. Research results from laboratory animals tend to support a cancer chemopreventive 
effect of tea components (Dreosti et al. 1997). However, the epidemiological studies are still somewhat inconclusive. It has been suggested that benefits from tea consumption are restricted to high intakes in high-risk populations (Kohlmeier et al. 1997). The consumption of five or more cups of green tea per day was shown to be associated with decreased recurrence of stage I and stage II breast cancer in Japanese woman (Nakachi et al. 1998).

\section{Broccoli and other cruciferous vegetables}

Epidemiological evidence has also associated the frequent consumption of cruciferous vegetables with decreased cancer risk. In a review of eighty-seven case-control studies, Verhoeven et al. (1996) demonstrated an inverse association between consumption of total brassica vegetables and cancer risk. The percentages of case-control studies showing an inverse association between consumption of cabbage, broccoli, cauliflower and Brussels sprouts and cancer risk were 70, 56, 67 and $29 \%$, respectively. The anticarcinogenic properties of cruciferous vegetables have been attributed to their relatively high content of glucosinolates (Verhoeven et al. 1997). Glucosinolates are a group of glycosides stored within cell vacuoles of all cruciferous vegetables. Myrosinase, an enzyme found in plant cells, converts these compounds to a variety of hydrolysis products, including isothiocyanates and indoles. While a wide variety of naturally occurring and synthetic isothiocyanates have been shown to prevent cancer in animals (Hecht, 1995), attention has been focused on a particular isothiocyanate isolated from broccoli, i.e. sulforaphane. Sulforaphane has been shown to be a good inducer of a particular phase II enzyme, quinone reductase. Indole-3-carbinol has received attention for its cancer chemopreventive properties, particularly of the mammary gland. In addition to the induction of phase I and phase II detoxification reactions, indole-3-carbinol may reduce cancer risk by modulating oestrogen metabolism.

\section{Citrus fruits}

Several epidemiological studies have shown that citrus fruits are protective against a variety of human cancers. Although oranges, lemons, limes and grapefruits are a principal source of such important nutrients as vitamin C, folate and fibre, Elegbede et al. (1993) have suggested that another component is responsible for the anticancer activity. Citrus fruits are particularly high in a class of phytochemicals known as the limonoids (Hasegawa \& Miyake, 1996). In recent years, evidence has been accumulating in support of the cancer-preventative effect of limonene (Gould, 1997). Crowell (1997) showed this compound to be effective against a variety of both spontaneous and chemically induced rodent tumours.

However, mindful of the importance of the overall dietary pattern in cancer risk reduction, one must question the clinical implications of a single phytochemical in isolation.

\section{Biomarkers available for assessing diet-related changes in colon cancer risk}

Since colon cancer has been a major target for the cancer chemopreventive effects of probiotics and functional food components, I shall limit my examples of 'biomarkers' to this cancer form. Presently, there are a large number of biomarkers available for assessing colon cancer risk in dietary intervention studies, which are validated to varying degrees. These include colonic mucosal markers, faecal water markers and immunological markers.

\section{Colon mucosa biomarkers}

1. Adhesion of Gram-negative bacteria

2. Modulation of cyclo-oxygenase-2 (COX-2)

3. Proliferation

4. K-ras

5. Genetic instability

6. Apoptosis

7. DNA-repair integrity

8. Metastasis markers

9. Microsatellite instability

10. Oxidative DNA damage

11. Gene-specific damage

\section{Faecal water markers}

In recent years, there has been considerable interest in the role of the aqueous phase of human faeces (faecal water) in studies examining the mechanisms underlying the dietary aetiology of colon cancer. The motivation is that components of this faecal fraction are more likely to be able to exert untoward effects on the cells of the colonic epithelium than components bound to food residues and the bacterial mass.

1. Cytotoxicity

2. COX-2 induction

3. Caspase induction

4. Calprotectin levels

5. Activator protein-1 activation

6. Bile acid levels

7. Effects on metastasis

8. Genotoxicity

9. Effects on cell metabolism

10. Gene induction

\section{Immunological and inflammatory response markers}

In the colon:

1. Suppression of COX-2 induction by pro-inflammatory cytokines

In blood:

1. Natural killer cells

2. Lymphocyte proliferation

3. Cytokines (interleukin-2, interleukin- $\beta$, tumour necrosis factor)

Thus, in designing a dietary intervention study/clinical trial 
to study anticancer effects (colon cancer) of functional foods, it is recommended to use as many of the above 'state-of-the-art' biomarkers as is feasible.

\section{Examples of studies addressing changes in biomarker response to a functional food}

\section{Shift from dairy product-rich to dairy product-free diet}

Cytotoxicity of faecal water is now an accepted risk marker for colon cancer and several studies have correlated toxicity of this faecal fraction with a higher colonic cell proliferation and increased colon cancer risk (Lapré \& Van der Meer, 1992). Genotoxicity per se in human faeces is also generally accepted as a risk marker for colon cancer. Recently, Pool-Zobel et al. (1996) have demonstrated, by employing the single cell gel electrophoresis (COMET) assay, that colon carcinogens induced genotoxicity in the colon of rats and that this genotoxicity was altered by dietary manipulations.

To determine whether the cytotoxicity and genotoxicity of the aqueous phase of human stools (faecal water) were affected by a change in dairy product intake, eighteen healthy male and female volunteers were randomly divided into two groups (Glinghammar et al. 1997). In a cross-over design, the volunteers shifted from their normal dairy product-rich to a dairy product-free diet. Nutritional analysis of the food consumed, during the study period, showed a significant decrease in energy intake from $9000 \mathrm{~kJ} / \mathrm{d}$ to $7866 \mathrm{~kJ} / \mathrm{d}(P=0.004)$, due to decreased intakes of protein and fat. Carbohydrate and fibre intakes remained unchanged during the intervention. $\mathrm{Ca}$ intake decreased significantly from $1488 \mathrm{mg} / \mathrm{d}$ to $372 \mathrm{mg} / \mathrm{d}$ $(P<0.001)$, with phosphate and vitamin $\mathrm{D}$ displaying similar significant decreases $(P<0.001)$. Cytotoxicity of faecal water, analysed by the HT-29 cytotoxicity assay, indicated a significant decrease $(P=0.025)$ in cell survival from $34 \%$ to $20 \%$ when dairy products were excluded from the participants' diet. This effect is most likely due to the decreased intake of dairy $\mathrm{Ca}$ and possibly phosphate. The COMET assay, used to analyse genotoxicity of faecal waters, indicated no differences brought about by the dietary intervention. These findings indicate that a shift from a dairy product-rich to a dairy product-free diet results in a significant effect $(P=0.025)$ on an accepted risk marker for colon cancer, and may suggest that the mechanism by which dairy products are protective is at the level of tumour promotion rather than initiation.

\section{Clinical trial to examine the effect of a synbiotic preparation on colon cancer risk biomarkers in adenoma patients}

Another example of such a study is the ongoing Symbiotics and Cancer Prevention in Humans (SYNCAN) project, funded by the EU, and involving eight research centres in Europe. It involves a twelve-week, randomised, double-blind placebo-controlled trial of a food supplement containing L. rhamnosus GG, Bifidobacterium Bb-12 and Raftilose Synergyl in adenoma patients. In this study, all of the colon cancer risk biomarkers, listed above, will be measured. In parallel, a long-term tumourigenesis study in rats, using the same synbiotic combination and assaying for the same biomarkers, is being carried out. It is hoped that the results of this study will provide much needed information on the cancer-protective effects of synbiotics in man and on the underlying mechanisms.

\section{References}

Biasco G, Paganelli GM, Brandi G, Brillanti S, Lami F, Callegari C \& Gizzi G (1991) Effect of Lactobacillus acidophilus and Bifidobacterium bifidum on rectal cell kinetics and fecal $\mathrm{pH}$. Italalian Journal of Gastroenterology 23, 142.

Block E (1992) The organosulfur chemistry of the genus Allium - implications for the organic chemistry of sulfur. Angewandte Chemie, International Edition Engl. 31, 1135-1178.

Block G, Patterson B \& Subar A (1992) Fruit, vegetables, and cancer prevention: a review of the epidemiological evidence. Nutrition and Cancer 18, 1-29.

Clinton SK (1998) Lycopene: chemistry, biology and implications for human health and disease. Nutrition Reviews 56, 35-51.

Crowell PL (1997) Monoterpenes in breast cancer chemoprevention. Breast Cancer Research and Treatment 46, 191-197.

Di Mascio P, Kaiser S \& Sies H (1989) Lycopene as the most efficient biological carotenoid singlet oxygen quencher. Archives Biochemistry and Biophysics 274, 532-538.

Dorant E, van den Brandt PA, Goldbohm RA, Hermus RJJ \& Sturmans F (1993) Garlic and its significance for the prevention of cancer in humans: a critical review. British Journal of Cancer 67, 424-429.

Dreosti IE, Wargovich MJ \& Yang CS (1997) Inhibition of carcinogenesis by tea: the evidence from experimental studies. Critical Reviews in Food Science and Nutrition 37, 761-770.

Elegbede JA, Maltzman TH, Elson CE \& Gould MN (1993) Effects of anticarcinogenic monoterpenes on phase II hepatic metabolizing enzymes. Carcinogenesis 14, 1221-1223.

Ernst E (1997) Can allium vegetables prevent cancer? Phytomedicine 4, 79-83.

Fuller R (1989) Probiotics in man and animals. Journal of Applied Bacteriology 66, 365-378.

Giovannucci E, Ascherio A, Rimm EB, Stampfer MJ, Colditz GA $\&$ Willett WC (1995) Intake of carotenoids and retinol in relation to risk of prostate cancer. Journal of the National Cancer Institute 87, 1767-1776.

Glinghammar B, Venturi M, Rowland IR \& Rafter J (1997) Shift from a dairy product-rich to a dairy product-free diet: influence on cytotoxicity and genotoxicity of fecal water - potential risk factors for colon cancer. American Journal of Clinical Nutrition 66, 1277-1282.

Gould MN (1997) Cancer chemoprevention and therapy by monoterpenes. Environmental Health Perspectives 105, 977-979.

Govers MJAP, Termont DSML, Lapré JA, Kleibeuker JH, Vonk RJ \& Van der Meer R (1996) Calcium in milk products precipitates intestinal fatty acids and secondary bile acids and thus inhibits colonic cytotoxicity in humans. Cancer Research 56, 3270-3275.

Graham HN (1992) Green tea composition, consumption and polyphenol chemistry. Preventive Medicine 21, 334-350.

Ha YL, Grimm NK \& Pariza MW (1987) Anticarcinogens from fried ground beef: health-altered derivatives of linoleic acid. Carcinogenesis 8, 1881-1887.

Hasegawa S \& Miyake M (1996) Biochemistry and biological 
functions of citrus limonoids. Food Reviews International 12, 413-435.

Hecht SS (1995) Chemoprevention by isothiocyanates. Journal of Cellular Biochemistry Supplement 22, 195-209.

Hirayama K \& Rafter J (2000) The role of probiotic bacteria in cancer prevention. Microbes and Infection 2, 681-686.

Intestinal Microbiology Group, International Agency for Research on Cancer (1977) Dietary fiber, transit time, faecal bacteria, steroids and colon cancer in two Scandinavian populations. Lancet 2, 207-211.

Ip C \& Scimeca JA (1997) Conjugated linoleic acid and linoleic acid are distinctive modulators of mammary carcinogenesis. Nutrition and Cancer 27, 131-135.

Iscovich JM, L Ábbé KA, Castelleto R, Calzona A, Bernedo A, Chopita NA, Jmelnitzsky AC \& Kaldor J (1992) Colon cancer in Argentina. Risk from dietary items. International Journal of Cancer 51, 851-857.

Kampman E, Giovannucci E, van't Veer P, Rimm E, Stampfer MJ, Colditz GA, Kok FJ \& Willett WC (1994a) Calcium, vitamin D, dairy foods, and the occurrence of colorectal adenomas among men and women in two prospective studies. American Journal of Epidemiology 139, 16-29.

Kampman E, Goldbohm RA, van den Brandt PA \& van't Veer P (1994b) Fermented dairy products, calcium, and colorectal cancer in the Netherlands cohort study. Cancer Research 54, 3186-3190.

Kohlmeier L, Weerings KGC, Steck S \& Kok FJ (1997) Tea and cancer prevention - an evaluation of the epidemiologic literature. Nutrition and Cancer 27, 1-13.

Lapre J, De Vries T, Termont DS, Kleibeuker J, De Vries EG \& Van der Meer R (1993) Mechanism of the protective effect of supplemental dietary calcium on cytolytic activity of fecal water. Cancer Research 53, 248-253.

Lapré JA \& Van der Meer R (1992) Diet-induced increase of colonic bile acids stimulates lytic activity of fecal water and proliferation of colonic cells. Carcinogenesis 13, 41-44.

Lidbeck A, Overvik E, Rafter J, Nord CE \& Gustafsson J-A (1992) Effect of Lactobacillus acidophilus supplements on mutagen excretion in feces and urine in humans. Microbial Ecology in Health and Disease 5, 59-67.
McKeown-Eyssen GE \& Bright-See E (1984) Dietary factors in colon cancer: international relationships. Nutrition and Cancer 6, 160-170.

Nakachi K, Suemasu K, Suga K, Takeo T, Imai K \& Higashi Y (1998) Influence of drinking green tea on breast cancer malignancy among Japanese patients. Japanese Journal of Cancer Research 89, 254-261.

Peters RK, Pike MC, Garabrant D \& Mack TM (1992) Diet and colon cancer in Los Angeles County, California. Cancer Causes and Control 3, 457-473.

Phipps WR, Martini MC, Lampe JW, Slavin JL \& Kurzer MS (1993) Effect of flax seed ingestion on the menstrual cycle. Journal of Clinical Endocrinology and Metabolism 77, $1215-1219$.

Pierre F (1997) Short-chain fructo-oligosaccharides reduce the occurrence of colon tumors and develop gut-associated lymphoid tissue in MIN mice. Cancer Research 57, 225-228.

Pool-Zobel BL, Neudecker C, Domizlaff I, Ji S, Schillinger U, Rumney CJ \& Rowland IR (1996) Lactobacillus and bifidobacterium-mediated antigenotoxicity in the colon of rats. Nutrition and Cancer 26, 365-380.

van't Veer P, Dekker JM, Lamers JWJ, Kok FJ, Schouten EG, Brants HAM, Sturmans F \& Hermus RJJ (1989) Consumption of fermented milk products and breast cancer: a case-control study in the Netherlands. Cancer Research 49, 4020-4023.

Verhoeven DT, Goldbohm RA, van Poppel G, Verhagen H \& van den Brandt PA (1996) Epidemiological studies on brassica vegetables and cancer risk. Cancer Epidemiology, Biomarkers and Prevention 5, 733-748.

Verhoeven DT, Verhagen H, Goldbohm RA, van den Brandt PA \& van Poppel G (1997) A review of mechanisms underlying anticarcinogenicity by brassica vegetables. Chemical Biological Interactions 28, 79-129.

Weisburger JH (1998) International Symposium on Lycopene and Tomato Products in Disease Prevention: an introduction. Proceedings of the Society of Experimental Biology and Medicine 218, 93-94.

Young TB \& Wolf D (1988) A case-control study of proximal and distal colon cancer and diet in Wisconsin. International Journal of Cancer 42, 167-175. 